\title{
LA LUCHA DE LAS MUJERES LATINOAMERICANAS, DEMOCRACIA Y DERECHOS HUMANOS
}

\section{Patricia Galeana*}

RESUMEN: Las mujeres latinoamericanas han participado activamente tanto en las luchas libertarias de nuestra América como en los procesos de integración. Pero su acción ha sido olvidada hasta el tiempo presente en que el feminismo ha abierto una nueva línea de investigación historiográfica. Las mujeres mantienen una "doble militancia", luchan por la igualdad para las mujeres y las transformaciones estructurales a favor de la soberanía nacional y regional y de la eliminación de las desigualdades económicas. Ciertamente esos ideales son utópicos, pero en el transcurso de la historia de la humanidad han sido las utopías las que han movido la parte más noble del espíritu humano.

PALABRAS CLAVE: Luchas sociales, Mujeres, Derechos humanos, Feminismo, Democracia.

\author{
[...] Una mujer superior \\ en elecciones no vota, \\ $y$ vota el pillo peor. \\ (Permitidme que me asombre.) \\ Con tal que aprenda a firmar \\ puede votar un idiota, \\ iporque es hombre!
}

Adela SAMUdio

Desde que en 1815 Simón Bolívar escribió su Carta de Jamaica para la conformación de la unión hispanoamericana, su ideal se convirtió en una utopía, aún insatisfecha hasta nuestros días. A lo largo de su histo-

* Facultad de Filosofía y Letras, UNAM (p_galeana@yahoo.com). 
ria, en las crisis, ante las amenazas del exterior, los países latinoamericanos se han dado muestras de solidaridad entre sí, pero sus problemas internos en el proceso de construcción de sus Estados nacionales les ha impedido llevar a cabo la plena unión latinoamericana. A pesar de los diversos proyectos de integración, ninguno de los modelos ha funcionado. No obstante, sus comunidades académicas sí se han mantenido en estrecha comunicación, al tiempo que ha habido avances en materia de constitución de bloques regionales en pro de la unión. ${ }^{1}$

Las mujeres latinoamericanas han participado activamente tanto en las luchas libertarias de nuestra América como en los procesos de integración. Sin embargo, su acción fue olvidada, hasta el tiempo presente, en que el feminismo ha abierto una nueva línea de investigación historiográfica. Baste como ejemplo, el que aún las máximas heroínas de la Independencia mexicana no cuentan con una biografía completa. Y las circunstancias de la casa de Leona Vicario, que por decreto del Congreso de la Unión de la etapa independiente se le otorgó en reconocimiento de su labor, y en vez de ser un museo dedicado a su memoria es hoy una oficina pública.

El venezolano Francisco de Miranda, precursor de la Independencia y pionero de la unidad latinoamericana, desde fines del siglo XVIII, planteó que las mujeres debían ser consultadas en las actividades políticas, pues "esa mitad de individuos tenía derecho a estar representada en el gobierno". Consideró a la marginación de la mujer "una injusticia irritante", que los legisladores americanos debían remediar. ${ }^{2}$ Miranda ya planteaba el derecho al voto de la mujer:

¿Por qué en un gobierno democrático, la mitad de los individuos no están directa o indirectamente representados, siendo así que ellas, las mujeres

${ }^{1}$ Por ejemplo, de Centroamérica, o desde el punto de vista económico con el establecimiento del Mercosur.

${ }^{2}$ Lucila Luciani de Pérez, "Miranda, precursor del feminismo", en Rev. Nac. de Cultura, núms. 78-79, Caracas, 1950, p. 27. 
se hallan igualmente sujetas a esa severidad de las leyes que los hombres han hecho conforme a su voluntad? ¿por qué, al menos, no se las consulta acerca de las leyes que les conciernen más directamente, como son las del matrimonio, divorcio, educación de los hijos. ${ }^{3}$

Desde que se consumó la independencia de sus países, las mujeres latinoamericanas del siglo XIX comienzan su lucha por sus derechos laborales, su derecho a la educación y su participación política.

Las revistas femeninas tienen un papel importante en la toma de conciencia de las mujeres. ${ }^{4}$ En México, en 1870 , en torno al periódico Siempre Viva, un grupo de mujeres se organizaron como Las hijas del Anáhuac, para exigir sus derechos. En 1883, Concepción Jimeno, fundadora de El Álbum de la mujer señalaba: "la mujer no es solamente un útero". En esa misma década se crea Violetas del Anáhuac por Laureana Wright Kleinhaus, que promueve la emancipación de la mujer a través de la educación; Juana Gutiérrez fundó Vésper y Amigas del pueblo e hijas de Cuauhtémoc, exigiendo derechos laborales y políticos. En 1901, el periodismo feminista tiene en Uruguay, con la revista Nosotras, un buen ejemplo de lucha. En 1902, Ana Roqué de Duprey, puertorriqueña, y Zoila Ugarte, ecuatoriana, son pioneras del periodismo femenino, las dos abogaron por el derecho a la educación y al sufragio. Ecuador y Puerto Rico fueron los primeros en otorgar el derecho al voto en 1929.

"La difusión del periodismo femenino es una necesidad imperiosa como medio de propagar la educación de la mujer y la causa del feminismo [...] eminentemente liberal, combatiendo los prejuicios y con-

${ }^{3}$ Carracciolo Parra Pérez, Miranda y la revolución francesa, Caracas, Ed. Culturales del Banco del Caribe, 1966, t. II, p. 67.

${ }^{4}$ En 1830, se publicó La Argentina, primer periódico de mujeres de América Latina, le siguió El Semanario de las Señoritas Mexicanas en 1836. En 1852, O Jornal das Senhoras en Brasil y en 1862, O Bello Sexo en Río de Janeiro. En las últimas décadas del siglo XIX, se fundan periódicos y revistas de mujeres en Bolivia, Colombia, Ecuador, Perú, Puerto Rico, Venezuela y México. 
vencionalismos arcaicos", escribe María F. Caminos durante el Primer Congreso Femenino Internacional, realizado en mayo de 1910 en Argentina. ${ }^{5}$ En el Congreso Feminista de Yucatán en 1916, así como en los movimientos feministas de los años treinta se luchó en Pro-Derechos de la Mujer en México. El Primer Congreso Feminista Panamericano, fue celebrado en Baltimore en 1922. ${ }^{6}$ Acudieron más de cien ponentes, la mayoría mexicanas. Aunque había diferencias políticas, se pugnaba por la igualdad y suprimir las diferencias de género. Se consideró entonces que había que acentuar algunas diferencias para exigir la igualdad en otros campos. Las principales conclusiones fueron: igualdad de Derechos Políticos; reforma a la Ley Electoral y representación parlamentaria. ${ }^{7}$

En México desde 1824 las zacatecanas habían exigido sus derechos políticos, pero tuvo que pasar siglo y medio para que se otorgara la

5 María F. Caminos, "Periodismo femenino", Buenos Aires, mayo de 1910.

${ }^{6}$ En la delegación mexicana estuvieron: Elena Torres, Eulalia Guzmán, Luz Vera, Aurora Herrera, María Rentería y Julia Nava.

${ }^{7}$ Igual criterio para el hombre y la mujer y reformas a los artículos constitucionales: $77,93,97$ y 101, igualdad civil, el juicio de divorcio termina en seis meses y que los hijos permanecieran con la madre. Los servicios de beneficencia debían estar en manos de las mujeres. Los colegios debían dar una enseñanza biológica y graduada. El control de la natalidad; cuidados prenatales y postnatales; y paternidad responsable, así como la formación de sociedades cooperativas, eran vistos como elementos para solucionar los problemas económicos.

En cuanto al trabajo, pidieron la misma retribución que el hombre, fomentar la creación de escuelas experimentales, servicios de salud. Reconocimiento del trabajo doméstico y creación de guarderías, así como Kindergartens populares.

Exigían que las mujeres fueran atendidas por mujeres en las comisarías. En cuanto a la migración: que en la costa y en las fronteras se orientara a las mujeres y efectuar una campaña moralizadora de la prensa.

Posteriormente, siguiendo la tradición feminista, en 1975, la guatemalteca, Alaide Foppa, funda la revista Fem, una revista feminista de "análisis, discusión y lucha que diera cabida a la creación literaria de mujeres que escriben con sentido feminista y que contribuyen con su obra al reconocimiento de ese nuevo ser, libre, independiente y productivo". Cfr. Consideraciones Primer Congreso Feminista Panamericano. 
ciudadanía a las mexicanas. Las mujeres chilenas aprovecharon que la Constitución de 1833 no establecía explícitamente la prohibición del voto femenino para exigirlo, pero no se reconoció su calidad de ciudadanas. Cuando años más tarde, el ministro chileno, Ignacio Zenteno sostuvo que a su juicio las mujeres podían y debían votar, porque la Constitución de Chile y la ley de 1874 les daba ese derecho, su propuesta fue considerada propia de un trastornado. En 1884, se negó de modo expreso en su artículo $4^{\circ}$ el voto a las mujeres chilenas. Mientras tanto, la Constitución liberal de 1886 en El Salvador otorgó la ciudadanía a sus mujeres. ${ }^{8}$

No obstante, en 1888, los antropólogos afirmaban:

el cerebro de la mujer pesa una décima parte menos que el del hombre [...] las diversas regiones no parecen igualmente desarrolladas [...] la mujer es un ser perpetuamente joven que debe colocarse entre el niño y el hombre (Letourneau) [...] Siendo el sufragio la manera directa de influir en el gobierno, preciso será averiguar si la mujer hará uso de él con entera independencia como lo exigen los principios de las instituciones representativas. ${ }^{9}$

La revolución liberal de 1895, liderada por Eloy Alfaro, aprobó decretos favorables a la mujer ecuatoriana. No obstante, Matilde Hidalgo, doctora en medicina de Ecuador, refiere que cuando el director del hospital donde trabajaba la despidió dijo: "Yo no trabajo con mujeres, déjese de andar metida en asuntos que sólo incumben a los hombres."

En México a pesar de la participación activa de las mujeres en la revolución, ésta no les hizo justicia y la Constitución de 1917 no las reconoció ciudadanas. En 1937, el presidente Lázaro Cárdenas congeló

${ }^{8}$ Según afirma Edelberto Torres-Rivas. Cfr. Interpretación del desarrollo social centroamericano, $2^{\text {a }}$ ed., Costa Rica, Universidad Centroamericana, 1971.

9 Luis López Méndez, "Los derechos políticos de la mujer” (diciembre de 1888), en Los partidos políticos, Caracas, Presidencia de la República, 1963. 
su propia iniciativa al dudar de su voluntad para votar independientemente de la Iglesia o de su esposo.

No puede existir la Democracia donde no tienen derechos políticos la mitad de la población. En México, este año conmemoramos apenas 50 años de ciudadanía de las mujeres. La división entre la vida pública y la privada, entre hombres y mujeres, ha obstaculizado el desarrollo de las democracias en nuestra América y en el mundo. ${ }^{10}$ Los dos siglos en que hemos estado inmersos en el liberalismo ha existido una contradicción entre la premisa libertad e igualdad individuales.

Las mujeres empezaron a cuestionar su condición de inferioridad al acceder a la educación. Pero por dos siglos se han seguido transmitiendo los valores que preservan la subordinación femenina, para que sean mejores madres y formar hijos para la "madre" Iglesia o ciudadanos para el Estado, sin importar su realización personal. La participación política permite el desarrollo de la capacidad de las mujeres como individuos. ${ }^{11}$ La independencia económica, proporciona seguridad personal y permite una ciudadanía participativa. El trinomio mujer, democracia y desarrollo es indivisible. ${ }^{12}$

En los años sesenta, los movimientos de liberación femenina coadyuvaron en la toma de conciencia de los distintos grupos oprimidos. Los movimientos de las mujeres han contribuido de manera importante a lograr una sociedad más justa, a la democratización de nuestros pueblos y a la defensa de sus derechos humanos. La mujeres han tenido una "doble militancia", luchan simultáneamente por la igual-

${ }^{10}$ Schumpeter no invalida que una organización política sea democrática, se apunta que la participación disminuyó. En el caso de Holanda, porque con la incorporación de la mujer, se pasó del voto obligatorio al voluntario.

${ }^{11}$ Fichte dice que la razón y la libertad son el fundamento de los derechos jurídicos. J. G. Fichte, The Science of Rights, trad. al inglés de A.E. Kroeger, London, Trubner, 1889, véase sobre todo el "Apéndice" (3.I.), p. 439.

${ }^{12}$ Locke creía que el poder paterno y el político son dependencia natural de los hijos respecto de sus padres. 
dad para las mujeres y las transformaciones estructurales, en favor de la soberanía nacional y regional y la eliminación de las desigualdades económicas.

Cuando en los años setenta aumenta el trabajo informal de las ciudades, ${ }^{13}$ las mujeres reiteran la demanda de salario igual para trabajo igual, así como autonomía sexual. Las dictaduras militares y guerras civiles de los años setenta y ochenta en varios países de la región latinoamericana revitalizaron, por su oposición, las luchas en pro de los derechos humanos, que no son sino códigos éticos que deben regir las relaciones entre las personas y el Estado. Los derechos humanos buscan la igualdad, son los instrumentos universalmente aceptados que hacen a los individuos sujetos de derechos, sujetos políticos, para la convivencia humana. ${ }^{14}$ Eric Hobsbaum, afirma en The Age of Capital que el modelo de familia burguesa es contradictorio con la nueva sociedad capitalista, al no regir la libertad y la igualdad de oportunidades. ${ }^{15}$

En los años ochenta, en Cuba ante la participación activa de las actividades económicas y políticas de las mujeres se obliga a los hombres a participar en los trabajos domésticos y la crianza de los hijos. Con esta medida se hace visible este trabajo para superar la "doble jornada", impuesta a las mujeres.

La ciudadanía de las mujeres ha sido fundamental para el cambio democrático, así como su impacto en el desarrollo de nuestra América. Pero subsiste la desigualdad de género, ${ }^{16}$ por lo que además del marco jurídico adecuado se requiere un sistema educativo eficiente que supere

13 Véase Helen Safa, "The Changing Class Competition of the Female Labour Force in Latin America", en Latin American Perspectives, vol. 4, núm. 4, 1977; Mariann Schmink, "La mujer en la economía en América Latina", documentos de trabajo del Consejo para la Población.

${ }^{14}$ Lourdes Arizpe, La mujer en el desarrollo de México y de América Latina, México, UNAM, 1989, pp. 25ss.

15 Ibid., p. 110.

${ }^{16}$ El concepto de género, denomina la construcción social de las identidades diferenciadas de mujeres y hombres. 
los atavismos patriarcales, ${ }^{17}$ así como campañas de difusión paralelas. La equidad significa igualdad de acuerdo con las diferencias. ${ }^{18}$ La evaluación de la actuación política de las mujeres es positiva, su participación en las urnas es elevada y su desempeño es mayoritariamente honesto y eficaz.

El proceso de globalización ha llevado a nuestros países a una dependencia, en lugar de una interdependencia, con un excesivo endeudamiento. En este contexto, crecen los riesgos para las democracias latinoamericanas, así como la falta de credibilidad en las instituciones políticas, empezando por los propios partidos. ${ }^{19}$ La participación de la mujer moraliza la política, lo que contribuye al fortalecimiento de la democracia.

A partir de 1975, las mujeres jefas de hogar pudieron ser titulares de parcelas ejidales en diversos países latinoamericanos. No obstante, las mujeres jóvenes siguen siendo enviadas al trabajo migratorio por no existir empleos para ellas en zonas rurales. "Las mujeres son más migrantes que los hombres". ${ }^{20}$ En América Latina y el Caribe, predo-

${ }^{17}$ La Ley del Instituto Nacional de las Mujeres fue publicada en el Diario Oficial de la Federación, el 12 de enero de 2001 y la Ley del Instituto de las Mujeres del Distrito Federal, se publicó en la Gaceta Oficial del Distrito Federal, el 28 de febrero de 2002 (núm. 28). Asimismo en diversas partes del mundo existen instituciones encargadas de vigilar el desarrollo de la mujer.

18 Prueba de lo anterior es la reciente recomendación en el mismo sentido del Banco Mundial, en la reunión de Dubai de septiembre pasado. El caso de Túnez es un ejemplo de lo que se puede desarrollar un pueblo a través de la educación de sus mujeres. En este país, el $80 \%$ de su población concluye la preparatoria y el $30 \%$ accede a la educación superior. En ella la mujer ocupa el 53.9\%. "Higher Profile for Women helps Boost the Economy", International Herald Tribune, sábado 27 de septiembre de 2003, p. 20.

${ }^{19}$ Situación que no es privativa de América Latina, sino que se está dando en diversos países del mundo.

${ }^{20}$ E. G. Ravenstein, 1885, “The Laws of Migration", en Journal of the Stalistical Society, XLVIII, part. II, en "Hacia una teoría de la migración femenina: La estructura social agraria y el éxodo de mujeres rurales en América Latina”, en Arizpe, op. cit., p. 217. 
minan las mujeres en la migración rural-urbana y, la población urbana femenina es mayor que la masculina en una proporción de 90:100. ${ }^{21}$ En los últimos treinta años el número de mujeres económicamente activas en América Latina y el Caribe creció en un 120.2\%. Sin embargo, se sigue teniendo el índice de participación femenina más bajo en la fuerza de trabajo comparado con Asia, África, Oceanía y países desarrollados.

El panamericanismo surgió como continuación del monroísmo. La ideología política de Estados Unidos hacia América Latina ${ }^{22}$ se apoyó en la relación asimétrica entre este país y los latinoamericanos, ${ }^{23}$ y se tradujo en su hegemonía sobre las distintas naciones del continente. De la declaración de la Doctrina Monroe en 1823 a la Primera Conferencia Panamericana, reunida en Washington en 1889, Estados Unidos propició la división entre los latinoamericanos, en oposición al hispanoamericanismo bolivariano, ${ }^{24}$ y buscó excluir a las potencias europeas de América. El panamericanismo planteó desde la última década del siglo XIX, la unión de todos los pueblos americanos, incluyendo a los caribeños, en torno a Estados Unidos. Posteriormente, en el siglo XX, en la segunda posguerra, la hegemonía estadounidense se institucionalizó en el sistema interamericano. No obstante, los latinoamericanos han pretendido reformar la Organización de Estados Americanos en diferentes acciones multilaterales concertadas para que responda a los intereses de todos sus miembros y no sólo a los de Estados Unidos. ${ }^{25}$

${ }^{21}$ CELADE, 1976, en "La participación de la mujer en el empleo y el desarrollo rural en América Latina y el Caribe: trabajo de síntesis", en ibid., pp. 109 ss.

22 José Luis Orozco, De teólogos, pragmáticos y geopolíticos. Aproximación al globalismo norteamericano, México, UNAM/Gedisa, 2001, pp. 223-233.

23 Alberto Díaz, "Panamericanismo", en Norberto Bobbio y Nicola Matucci [dirs.], Diccionario de Política, México, Siglo XXI, 1986, p. 1161.

${ }^{24}$ Francisco Cuevas Cancino, Del Congreso de Panamá a la Conferencia de Caracas 1826-1954. El genio de Bolívar a través de la historia de las relaciones interamericanas, Caracas, Ragon, 1955, t. I, pp. 221-272.

${ }^{25}$ Edmundo Hernández-Vela Salgado, Diccionario de Política Internacional, $3^{\text {a }}$ ed., México, Porrúa, 1988, pp. 119 y 190. 
Bolívar advirtió que "en la marcha de los siglos podría encontrarse, quizá, una sola nación cubriendo el universo". Creyó arar en el mar, al no lograr la conformación de la Patria de Patrias, la Patria Grande, la latinoamericana. Ambicionó la integración de América Latina, pero una integración entre pares, ya que de otra forma, alertó el libertador, “formando una vez el pacto con el fuerte, ya es eterna la obligación del débil".

Una gran mujer, Gabriela Mistral, alzó su voz contra las fuerzas armadas de un Estado gendarme que pretendiera dirigir al mundo; y clamó porque no se rebajara la libertad del espíritu a la servidumbre. Es menester tener presentes sus postulados cuando el país hegemónico del mundo global presenta a su seguridad nacional como prioritaria, por encima de nuestros principios soberanos, de la democracia y de los derechos humanos.

Ciertamente esos ideales son utópicos, pero a lo largo de la historia de la humanidad, han sido las utopías las que han movido la parte más noble del espíritu humano. 